City University of New York (CUNY) CUNY Academic Works

1989

\title{
Topological Gauge Theory and Twistors
}

V. Parameswaran Nair

City College of New York

Jeremy Schiff

Columbia University

\section{How does access to this work benefit you? Let us know!}

More information about this work at: https://academicworks.cuny.edu/cc_pubs/373

Discover additional works at: https://academicworks.cuny.edu

This work is made publicly available by the City University of New York (CUNY).

Contact: AcademicWorks@cuny.edu 
CU-TP-439

July 1989

\title{
Topological Gauge Theory and Twistors
}

\author{
V.P.Nair and Jeremy Schiff \\ Physics Department, Columbia University \\ New York, NY 10027
}

\begin{abstract}
By use of harmonic superfields, Witten's topological gauge theory is written as a field theory on a supertwistor space. Further, using the realisation of complex compactified Minkowski space as a quadric in $\mathbf{C} P^{5}$, we show that the theory has a simple formulation in terms of analytic superfields on super- $\mathbf{C} P^{5} \times \mathbf{C} P^{3}$.
\end{abstract}

This research was supported in part by the U.S. Department of Energy 


\section{Introduction}

In this paper we give a twistor theoretic description of the topological gauge theory constructed by Witten, in which the Donaldson polynomial invariants of four-manifolds can be obtained as correlation functions [1],[2]. Several authors have observed that Witten's action is a gauge fixed version of a topological action $\int \operatorname{Tr}(F \wedge F)$, or something similar, through a BRST procedure [3]. In [1] Witten suggested another interpretation of the theory, at least on flat space, as an $N=2$ supersymmetric gauge theory with a reinterpretation of the action of the Lorentz group. The internal $S U(2)_{I}$ relating the two supersymmetries is identified with $S U(2)_{R}$, the right chirality component of the Lorentz group. We shall develop this point of view using the harmonic superspace approach to $N=2$ supersymmetric gauge theory [4]. In this approach one considers superfields not on regular superspace $M$ but on harmonic superspace which is of the form $M \times \mathbf{C} P^{1}$. The authors of [4] show that the constraints of $N=2$ supersymmetric gauge theory can be solved in terms of analytic fields on harmonic superspace, giving an unconstrained superfield formalism for the theory. With the reinterpretation of the action of the Lorentz group, we obtain a superfield description of the Witten theory (distinct from the superfield formulation given in [5]), defined on a supersymmetric version of twistor space [6]. The BRST-type Q-symmetry used in proving the topological invariance of the theory becomes a Grassman analogue of the scaling symmetry $\pi^{\dot{\alpha}} \rightarrow \lambda \pi^{\dot{\alpha}}, \lambda \in \mathbf{C}$ which allows us to go from $\mathbf{C}^{2}-\{0\}$ defined by $\pi^{\dot{\alpha}}, \dot{\alpha}=0,1$, to the projective space $\mathbf{C} P^{1}$.

Another geometrical description of spacetime, closely related to twistor theory, is the $\mathbf{C} P^{5}$ calculus [6], [7]. Complex compactified Minkowski space can be realised as a quadric $\Omega(X)=0$ in $\mathbf{C} P^{5}, X^{M}, M=1, \ldots, 6$ being homogeneous coordinates for $\mathbf{C} P^{5}$. We show that topological gauge theory has a very natural formulation in this language. In particular the Lagrangian is given by $\operatorname{Tr}\left(A_{\Omega}\right)^{2}$ where $A_{\Omega}$ is the (Lie algebra valued) gauge potential along the direction $\frac{\partial}{\partial \Omega}$ orthogonal to $\Omega(X)=0$, i.e. orthogonal to (complex) Minkowski space. The relationship with twistor space can be recovered by the well-known observation that certain lines in twistor space define (complex) Minkowski space, and conversely certain planes in Minkowski space define twistor space [6]. The $\mathbf{C} P^{5}$ description also extends 
naturally to conformally flat manifolds.

A suggestion that twistor space may be a natural setting for topological field theory was also made recently by Penrose [8]. In the particular case of topological gauge theory, the theory gives information about instanton moduli space, and via the Ward correspondence, instantons correspond to certain holomorphic vector bundles on twistor space [9]. Thus we feel that the twistor formulation may give some extra insight into topological gauge theory.

\section{Harmonic Superspace Formalism}

We begin with a description of the harmonic superfield formalism of $N=2$ gauge theory. Spinor notation will be used, i.e. $\psi^{\alpha}, \psi^{\dot{\alpha}}$ transform as spinors of $S U(2)_{L}$ and $S U(2)_{R}$ respectively, which are left and right components of the Lorentz group. We use the conventions of [4] for raising and lowering of indices. Harmonic superspace is described by coordinates $\left(x^{\alpha \dot{\alpha}}, \theta_{i}^{\alpha}, \bar{\theta}^{\dot{\alpha} i}, \pi^{i}\right)(i=0,1)$ where the $\theta^{\prime}$ s are Grassman variables and $\pi^{i}$ are two complex variables, $\pi^{0}=\pi^{1}=0$ excluded, but the theory has invariance under $\pi^{i} \rightarrow \lambda \pi^{i}$, so that ultimately the $\pi^{\prime}$ 's describe $\mathbf{C} P^{1}$. Alternatively one can use $u^{i}=\frac{\pi^{i}}{\sqrt{|\pi|^{2}}}$ which describe $S U(2)$. Invariance under $U(1) \subset S U(2)$ can be achieved by assigning a $U(1)$ charge +1 to $u^{i}$ and -1 to $\bar{u}^{i}=\left(-\left(u^{2}\right)^{*},\left(u^{1}\right)^{*}\right)$ and ensuring balance of these charges in any equation (here ${ }^{*}$ denotes complex conjugation).

We define $\xi^{\alpha}=\theta_{i}^{\alpha} u^{i}, \bar{\xi}^{\alpha}=\theta_{i}^{\alpha} \bar{u}^{i}, \eta^{\dot{\alpha}}=\bar{\theta}_{i}^{\dot{\alpha}} u^{i}, \bar{\eta}^{\dot{\alpha}}=\bar{\theta}_{i}^{\dot{\alpha}} \bar{u}^{i}$ and consider the following basis of vector fields on harmonic superspace:

$$
\begin{gathered}
D_{\alpha \dot{\alpha}}=\frac{\partial}{\partial x^{\alpha \dot{\alpha}}} \\
D_{\alpha}^{+}=\frac{\partial}{\partial \bar{\xi}^{\alpha}}-\frac{1}{2} \eta^{\dot{\alpha}} D_{\alpha \dot{\alpha}} \\
D_{\dot{\alpha}}^{+}=\frac{\partial}{\partial \bar{\eta}^{\dot{\alpha}}}+\frac{1}{2} \xi^{\alpha} D_{\alpha \dot{\alpha}} \\
D_{\alpha}^{-}=\frac{\partial}{\partial \xi^{\alpha}}+\frac{1}{2} \bar{\eta}^{\dot{\alpha}} D_{\alpha \dot{\alpha}} \\
D_{\dot{\alpha}}^{-}=\frac{\partial}{\partial \eta^{\dot{\alpha}}}-\frac{1}{2} \bar{\xi}^{\alpha} D_{\alpha \dot{\alpha}}
\end{gathered}
$$




$$
\begin{gathered}
D^{++}=u^{i} \frac{\partial}{\partial \bar{u}^{i}}+\xi^{\alpha} \frac{\partial}{\partial \bar{\xi}^{\alpha}}+\eta^{\dot{\alpha}} \frac{\partial}{\partial \bar{\eta}^{\dot{\alpha}}} \\
D^{--}=\bar{u}^{i} \frac{\partial}{\partial u^{i}}+\bar{\xi}^{\alpha} \frac{\partial}{\partial \xi^{\alpha}}+\bar{\eta}^{\dot{\alpha}} \frac{\partial}{\partial \eta^{\dot{\alpha}}} \\
D^{0}=\frac{1}{2}\left(u^{i} \frac{\partial}{\partial u^{i}}+\xi^{\alpha} \frac{\partial}{\partial \xi^{\alpha}}+\eta^{\dot{\alpha}} \frac{\partial}{\partial \eta^{\dot{\alpha}}}-\bar{u}^{i} \frac{\partial}{\partial \bar{u}^{i}}-\bar{\xi}^{\alpha} \frac{\partial}{\partial \bar{\xi}^{\alpha}}-\bar{\eta}^{\dot{\alpha}} \frac{\partial}{\partial \bar{\eta}^{\dot{\alpha}}}\right)
\end{gathered}
$$

Here the +'s and -'s associated with each operator tell us its associated $U(1)$ charge. Any expression of fixed charge is an eigenfunction of $D^{0}$ (with eigenvalue half of the charge), so for our purposes $D^{0}$ can be regarded as a number, rather than a differential operator. We note the following graded commutation relations of the above operators:

$$
\begin{gathered}
\left\{D_{\alpha}^{+}, D_{\beta}^{+}\right\}=\left\{D_{\dot{\alpha}}^{+}, D_{\dot{\beta}}^{+}\right\}=\left\{D_{\alpha}^{+}, D_{\dot{\alpha}}^{+}\right\}=0 \\
\left\{D_{\alpha}^{-}, D_{\beta}^{-}\right\}=\left\{D_{\dot{\alpha}}^{-}, D_{\dot{\beta}}^{-}\right\}=\left\{D_{\alpha}^{-}, D_{\dot{\alpha}}^{-}\right\}=0 \\
\left\{D_{\alpha}^{-}, D_{\dot{\alpha}}^{+}\right\}=-\left\{D_{\alpha}^{+}, D_{\dot{\alpha}}^{-}\right\}=D_{\alpha \dot{\alpha}} \\
{\left[D^{++}, D_{\alpha}^{+}\right]=\left[D^{++}, D_{\dot{\alpha}}^{+}\right]=0} \\
{\left[D^{++}, D_{\alpha}^{-}\right]=-D_{\alpha}^{+}} \\
{\left[D^{++}, D_{\dot{\alpha}}^{-}\right]=-D_{\dot{\alpha}}^{+}} \\
{\left[D^{--}, D_{\alpha}^{-}\right]=\left[D^{--}, D_{\dot{\alpha}}^{-}\right]=0} \\
{\left[D^{--}, D_{\alpha}^{+}\right]=-D_{\alpha}^{-}} \\
{\left[D^{--}, D_{\dot{\alpha}}^{+}\right]=-D_{\dot{\alpha}}^{-}} \\
{\left[D^{++}, D^{--}\right]=2 D^{0}}
\end{gathered}
$$

These derivative operators can be made covariant by introducing gauge potentials for each of them, e.g. $D^{++} \rightarrow \nabla^{++}=D^{++}+A^{++}$, (we do not gauge $D^{0}$ though), and we can define field strengths in the obvious way, e.g. $F^{++,--}=\left[\nabla^{++}, \nabla^{--}\right]-2 D^{0}$. Now, given an $N=2$ gauge field on regular superspace $\left(\mathcal{A}_{\alpha \dot{\alpha}}, \mathcal{A}_{\alpha}^{i}, \mathcal{A}_{\dot{\alpha} i}\right)$ satisfying the usual constraints [10], we can define a gauge field on harmonic superspace by setting

$$
A^{++}=A^{--}=0
$$




$$
\begin{aligned}
& A_{\alpha}^{+}=\mathcal{A}_{\alpha}^{i} u^{i} \\
& A_{\alpha}^{-}=\mathcal{A}_{\alpha}^{i} \bar{u}^{i} \\
& A_{\dot{\alpha}}^{+}=\mathcal{A}_{\dot{\alpha}}^{i} u^{i} \\
& A_{\dot{\alpha}}^{-}=\mathcal{A}_{\dot{\alpha}}^{i} \bar{u}^{i} \\
& A_{\alpha \dot{\alpha}}=\mathcal{A}_{\alpha \dot{\alpha}}
\end{aligned}
$$

The field strengths associated with all the commutators in (2) will be zero by virtue of these assignments and the $N=2$ constraints. The usual $N=2$ field strengths $W, \bar{W}$ are given by

$$
\begin{aligned}
& \left\{\nabla_{\alpha}^{+}, \nabla_{\beta}^{-}\right\}=W \epsilon_{\alpha \beta} \\
& \left\{\nabla_{\dot{\alpha}}^{+}, \nabla_{\dot{\beta}}^{-}\right\}=\bar{W} \epsilon_{\dot{\alpha} \dot{\beta}}
\end{aligned}
$$

By virtue of the vanishing of the field strengths associated with the commutators in (2a) we can obtain a gauge equivalent description with $A_{\alpha}^{+}=A_{\dot{\alpha}}^{+}=0$. In this gauge $A^{++}$and $A^{--}$ will be non-zero. The vanishing of the field strengths associated with the commutators in $(2 \mathrm{~d}, \mathrm{~h}, \mathrm{i}, \mathrm{j}, \mathrm{c})$ in this gauge imply (respectively)

$$
\begin{gathered}
D_{\alpha}^{+} A^{++}=D_{\dot{\alpha}}^{+} A^{++}=0 \\
A_{\alpha}^{-}=D_{\alpha}^{+} A^{--} \\
A_{\dot{\alpha}}^{-}=D_{\dot{\alpha}}^{+} A^{--} \\
D^{++} A^{--}-D^{--} A^{++}+\left[A^{++}, A^{--}\right]=0 \\
A_{\alpha \dot{\alpha}}=D_{\dot{\alpha}}^{+} D_{\alpha}^{+} A^{--}
\end{gathered}
$$

Equation (5a) is the definition of analyticity in harmonic superspace; it requires that $A^{++}$ depends on $x^{\alpha \dot{\alpha}}, \bar{\xi}^{\alpha}, \bar{\eta}^{\dot{\alpha}}$ only through the combination $y^{\alpha \dot{\alpha}}=x^{\alpha \dot{\alpha}}+\frac{1}{2}\left(\xi^{\alpha} \bar{\eta}^{\dot{\alpha}}+\bar{\xi}^{\alpha} \eta^{\dot{\alpha}}\right)$. (5d) determines $A^{--}$in terms of $A^{++}$(see [11]), whence (5b,c,e) give all other components of the gauge field. Thus all physical quantities are given in terms of the analytic superfield $A^{++}$. (Furthermore it is possible to show that apart from the analyticity condition there are no other constraints on $A^{++}$; to do this, given an analytic $A^{++}$we construct a full 
gauge field on harmonic superspace by (5b)-(5e), and setting $A_{\alpha}^{+}=A_{\dot{\alpha}}^{+}=0$, then change gauge back to a gauge where $A^{++}=A^{--}=0$, using $(5 \mathrm{~d})$. From the gauge field in this gauge it is easy to construct a gauge field on regular superspace and to check it satisfies the usual $N=2$ constraints.) In the new gauge the field strengths are

$$
\begin{aligned}
& W=\frac{1}{2} D_{\alpha}^{+} D^{+\alpha} A^{--} \\
& \bar{W}=\frac{1}{2} D_{\dot{\alpha}}^{+} D^{+\dot{\alpha}} A^{--}
\end{aligned}
$$

These satisfy covariant chirality conditions so that

$$
\begin{aligned}
& D_{\alpha}^{+} \operatorname{Tr}\left(W^{2}\right)=D_{\alpha}^{-} \operatorname{Tr}\left(W^{2}\right)=0 \\
& D_{\dot{\alpha}}^{+} \operatorname{Tr}\left(\bar{W}^{2}\right)=D_{\dot{\alpha}}^{-} \operatorname{Tr}\left(\bar{W}^{2}\right)=0
\end{aligned}
$$

The action for $N=2$ theory can be taken as the sum of

$$
\begin{aligned}
& S_{+}=\int d^{4} x d u d^{2} \eta d^{2} \bar{\eta} \operatorname{Tr}\left(W^{2}\right) \\
& S_{-}=\int d^{4} x d u d^{2} \xi d^{2} \bar{\xi} \operatorname{Tr}\left(\bar{W}^{2}\right)
\end{aligned}
$$

where $d u$ is an invariant measure on $\mathbf{C} P^{1}=S U(2) / U(1)$.

In the formalism we have given the supersymmetry generators are given by

$$
\begin{gathered}
Q_{\alpha}^{i}=u^{i} \frac{\partial}{\partial \xi^{\alpha}}+\bar{u}^{i} \frac{\partial}{\partial \bar{\xi}^{\alpha}}+\frac{1}{2}\left(\bar{\eta}^{\dot{\alpha}} u^{i}-\eta^{\dot{\alpha}} \bar{u}^{i}\right) D_{\alpha \dot{\alpha}} \\
Q_{\dot{\alpha} j}=-u_{j} \frac{\partial}{\partial \eta^{\dot{\alpha}}}-\bar{u}_{j} \frac{\partial}{\partial \bar{\eta}^{\dot{\alpha}}}+\frac{1}{2}\left(\bar{\xi}^{\alpha} u_{j}-\xi^{\alpha} \bar{u}_{j}\right) D_{\alpha \dot{\alpha}}
\end{gathered}
$$

All of these (anti)commute with all of the derivatives in (1). Finally we note that in this formalism we can write $A^{++}$, following [3], in a Wess- Zumino type gauge in the form

$A^{++}=\phi(y) \eta \cdot \eta+\lambda(y) \xi \cdot \xi+V_{\alpha \dot{\alpha}}(y) \xi^{\alpha} \eta^{\dot{\alpha}}+\chi_{\dot{\alpha} i}(y) \xi \cdot \xi \eta^{\dot{\alpha}} \bar{u}^{i}+\psi_{\alpha i}(y) \eta \cdot \eta \xi^{\alpha} \bar{u}^{i}+D_{(i j)}(y) \xi \cdot \xi \eta \cdot \eta \bar{u}^{i} \bar{u}^{j}$

(where $\eta \cdot \eta=\eta_{\dot{\alpha}} \eta^{\dot{\alpha}}$ etc.) thus identifying the usual component fields of $N=2$ gauge theory $\left(D_{(i j)}\right.$ is an auxiliary field). 


\section{Topological Gauge Theory}

To obtain Witten's topological gauge theory, we identify $S U(2)_{I}$, under which $u^{i}=$ $\frac{\pi^{i}}{\sqrt{|\pi|^{2}}}$ is a doublet, with the right chirality component of the the Lorentz group, so we replace $\pi^{i}$ by $\pi^{\dot{\alpha}}$. Harmonic superspace is now given by coordinates $x^{\alpha \dot{\alpha}}, \pi^{\dot{\alpha}}, \bar{\pi}^{\dot{\alpha}}, \xi^{\alpha}, \bar{\xi}^{\alpha}, \eta^{\dot{\alpha}}, \bar{\eta}^{\dot{\alpha}}$. Projective twistor space, realised as a $\mathbf{C} P^{1}$ bundle over spacetime, is described by $\left(x^{\alpha \dot{\alpha}}, \pi^{\dot{\alpha}}, \bar{\pi}^{\dot{\alpha}}\right)$, so harmonic superspace is now a supersymmetric version of projective twistor space. The supertwistors thus obtained are related to, but not the same as, the standard supertwistors obtained from superextensions of the conformal group [12].

Because of the chirality property $(7 a), \operatorname{Tr}\left(W^{2}\right)$ can only depend on $x^{\alpha \dot{\alpha}}, \xi^{\alpha}, \bar{\xi}^{\alpha}$ through the combination $z^{\alpha \dot{\alpha}}=x^{\alpha \dot{\alpha}}+\frac{1}{2}\left(\bar{\xi}^{\alpha} \eta^{\dot{\alpha}}-\xi^{\alpha} \bar{\eta}^{\dot{\alpha}}\right)$. Defining $\omega^{\alpha}=z^{\alpha \dot{\alpha}} \pi_{\dot{\alpha}}, \bar{\omega}^{\alpha}=z^{\alpha \dot{\alpha}} \bar{\pi}_{\dot{\alpha}}$, we can write the action of the topological gauge theory as

$$
S_{+}=\int \frac{d^{2} \omega d^{2} \bar{\omega} d^{2} \pi d^{2} \bar{\pi} d^{2} \eta d^{2} \bar{\eta}}{(\pi \cdot \bar{\pi})^{2}} \operatorname{Tr}\left(W^{2}\right)
$$

Unlike $N=2$ gauge theory we do not include the hermitian conjugate $S_{-}$since (11) has $\left(F_{\mu \nu}-\tilde{F}_{\mu \nu}\right)^{2}, \tilde{F}_{\mu \nu}=\frac{1}{2} \epsilon_{\mu \nu \rho \sigma} F^{\rho \sigma}$, which is appropriate for instantons. (Needless to say, a conjugate description is obtained by identifying $S U(2)_{I}$ with $S U(2)_{L}$ and using $S_{-}$as the action).

We identify components in the Wess-Zumino gauge, as above, where now

$$
\begin{gathered}
A^{++}=\phi(y) \eta \cdot \eta+\lambda(y) \xi \cdot \xi+V_{\alpha \dot{\alpha}}(y) \xi^{\alpha} \eta^{\dot{\alpha}}+\chi_{\dot{\alpha} \dot{\beta}}(y) \xi \cdot \xi \eta^{\dot{\alpha}} \bar{u}^{\dot{\beta}}+\psi_{\alpha \dot{\alpha}}(y) \eta \cdot \eta \xi^{\alpha} \bar{u}^{\dot{\alpha}} \\
+D_{(\dot{\alpha} \dot{\beta})}(y) \xi \cdot \xi \eta \cdot \eta \bar{u}^{\dot{\alpha}} \bar{u}^{\dot{\beta}}
\end{gathered}
$$

This gives exactly the field content of [1] (we write $\chi_{\dot{\alpha} \dot{\beta}}=\eta(y) \epsilon_{\dot{\alpha} \dot{\beta}}+\chi_{(\dot{\alpha} \dot{\beta})}(y)$, giving Witten's fermionic scalar and fermionic antisymmetric tensor), with the auxiliaries $D_{(\dot{\alpha} \dot{\beta})}$. The Q-symmetry operator is given by $\epsilon^{\dot{\alpha} \dot{\beta}} Q_{\dot{\alpha} \dot{\beta}}$, where $Q_{\dot{\alpha} \dot{\beta}}$ are obtained from (9b). This gives

$$
Q=u \cdot \frac{\partial}{\partial \eta}+\bar{u} \cdot \frac{\partial}{\partial \bar{\eta}}+\frac{1}{2}\left(\bar{\xi}^{\alpha} u^{\dot{\alpha}}-\xi^{\alpha} \bar{u}^{\dot{\alpha}}\right) D_{\alpha \dot{\alpha}}
$$

The component action of the Q-symmetry can be obtained by letting $Q$ act on $A^{++}$, and then performing a gauge transformation $A^{++} \rightarrow g^{-1} A^{++} g+g^{-1} D^{++} g$ ( $g$ analytic) to restore Wess-Zumino gauge. 
Being a superfield formulation, it is trivial to see that the Lagrangian is Q-exact, i.e. is the Q-variation of another field. Writing

$$
\operatorname{Tr}\left(W^{2}\right)=\mathcal{L}(z) \eta \cdot \eta \bar{\eta} \cdot \bar{\eta}+V_{\dot{\alpha}}(z, u, \bar{u}) \eta^{\dot{\alpha}} \bar{\eta} \cdot \bar{\eta}+\bar{V}_{\dot{\alpha}}(z, u, \bar{u}) \bar{\eta}^{\dot{\alpha}} \eta \cdot \eta+\ldots
$$

we have

$$
Q \operatorname{Tr}\left(W^{2}\right)=2 \mathcal{L}(z) u \cdot \eta \bar{\eta} \cdot \bar{\eta}+2 \mathcal{L}(z) \bar{u} \cdot \bar{\eta} \eta \cdot \eta+\ldots
$$

So

$$
\begin{aligned}
& \delta_{Q} V_{\dot{\alpha}}=2 \mathcal{L} u_{\dot{\alpha}} \\
& \delta_{Q} \bar{V}_{\dot{\alpha}}=2 \mathcal{L} \bar{u}_{\dot{\alpha}}
\end{aligned}
$$

and thus we conclude $\mathcal{L}=\delta_{Q} V$ where $V=\frac{1}{2}\left(\bar{V}_{\dot{\alpha}} u^{\dot{\alpha}}-V_{\dot{\alpha}} \bar{u}^{\dot{\alpha}}\right)$. (An explicit formula for $\mathrm{V}$ in component fields, corresponding to that in [1], can easily be obtained, starting from the component form of $\mathrm{W}$ given in [10] for the regular $N=2$ theory. With the auxiliary fields, as expected, this proof does not depend on the equations of motion.)

It is also straightforward in this formalism to write the correlators yielding the Donaldson polynomials. From the chirality property $(7 b), \operatorname{Tr}\left(\bar{W}^{2}\right)$ only depends on $x^{\alpha \dot{\alpha}}, \eta^{\dot{\alpha}}, \bar{\eta}^{\dot{\alpha}}$ through the combination $\tilde{z}^{\alpha \dot{\alpha}}=x^{\alpha \dot{\alpha}}-\frac{1}{2}\left(\bar{\xi}^{\alpha} \eta^{\dot{\alpha}}-\xi^{\alpha} \bar{\eta}^{\dot{\alpha}}\right)$, and hence we can check that

$$
Q \operatorname{Tr}\left(\bar{W}^{2}\right)=\left(\bar{\xi}^{\alpha} u^{\dot{\alpha}}-\xi^{\alpha} \bar{u}^{\dot{\alpha}}\right) D_{\alpha \dot{\alpha}} \operatorname{Tr}\left(\bar{W}^{2}\right)
$$

and thus $\left.\operatorname{Tr}\left(\bar{W}^{2}\right)\right|_{\xi=\bar{\xi}=0}$ is Q-invariant (and gauge invariant). This is exactly the $\operatorname{Tr} \phi^{2}$ field used to construct the Donaldson polynomials, which can thus be identified with the correlators

$$
\left.\int d \mu\left(A^{++}\right) \exp \left(-S_{+}\right) \operatorname{Tr}\left(\bar{W}^{2}\right)\left(x_{1}\right) \operatorname{Tr}\left(\bar{W}^{2}\right)\left(x_{2}\right) \ldots \operatorname{Tr}\left(\bar{W}^{2}\right)\left(x_{n}\right)\right|_{\xi=\bar{\xi}=0}
$$

If throughout our work we had opted to work with the $\pi^{\dot{\alpha}}$ variables in place of the $u^{\dot{\alpha}}$ ones, and had defined $\tilde{\eta}^{\dot{\alpha}}=\bar{\theta}_{\dot{\beta}}^{\dot{\alpha}} \pi^{\dot{\beta}}\left(=\sqrt{|\pi|^{2}} \eta^{\dot{\alpha}}\right)$, then clearly the Lagrangian $\operatorname{Tr}\left(W^{2}\right)$ would be invariant under the scaling $\pi^{\dot{\alpha}} \rightarrow \lambda \pi^{\dot{\alpha}}, \tilde{\eta}^{\dot{\alpha}} \rightarrow \lambda \tilde{\eta}^{\dot{\alpha}}, \lambda \in \mathbf{C}$. Ignoring changes in $\operatorname{Tr}\left(W^{2}\right)$ which are total derivatives, the Q-symmetry is effectively $\tilde{\eta}^{\dot{\alpha}} \rightarrow \tilde{\eta}^{\dot{\alpha}}+\epsilon \pi^{\dot{\alpha}}$, where $\epsilon$ is a real Grassman number. If we write

$$
\Pi=\left(\begin{array}{cc}
\pi^{\dot{\alpha}} & \tilde{\eta}^{\dot{\alpha}} \\
0 & \pi^{\dot{\alpha}}
\end{array}\right)
$$


then we can combine the two symmetries as

$$
\Pi \rightarrow \Lambda \Pi, \quad \Lambda=\left(\begin{array}{cc}
\lambda & \epsilon \\
0 & \lambda
\end{array}\right)
$$

Q-symmetry is thus a Grassman analogue of the scaling $\pi^{\dot{\alpha}} \rightarrow|\lambda| \pi^{\dot{\alpha}}$. Since $\epsilon$ is taken to be a real Grassman number the phase information of $\lambda$ has no analogue.

\section{CP $P^{5}$ Formalism}

We shall now reformulate the theory in (super) $\mathbf{C} P^{5} \times \mathbf{C} P^{3}$. Introduce homogeneous coordinates for $\mathbf{C} P^{5}, X^{M}, M=1, \ldots, 6$, homogeneous coordinates for $\mathbf{C} P^{3}$, $Z^{i}=\left(\omega^{\alpha}, \pi^{\dot{\alpha}}\right), i=1, \ldots, 4$, and Grassman coordinates $Q^{i}=\left(\xi^{\alpha}, \eta^{\dot{\alpha}}\right), i=1, \ldots, 4$ (note the use of the index $i$ is now different from that of section 2). We can equivalently represent $X^{M}$ as an antisymmetric bitwistor $X^{i j}$ via

$$
X^{i j}=\left[\begin{array}{cc}
i \zeta \epsilon & x^{\mu} e^{\mu} \\
-x^{\mu}\left(e^{\mu}\right)^{T} & i \tilde{\zeta} \epsilon
\end{array}\right]
$$

where $\zeta=\frac{X^{5}-X^{6}}{\sqrt{2}}, \tilde{\zeta}=\frac{X^{5}+X^{6}}{\sqrt{2}}, x^{\mu}=X^{\mu}, e^{\mu}=\left(1, i \sigma^{k}\right)$ where $\sigma^{k}$ are the Pauli matrices, $\epsilon=i \sigma^{2}$ and $\mu$ is an index that runs from 1 to 4 (we shall also use the notation, as throughout this paper, $\left.x^{\alpha \dot{\alpha}}=\left(x^{\mu} e^{\mu}\right)^{\alpha \dot{\alpha}}\right)$. We inroduce the following derivatives:

$$
\begin{gathered}
D_{i j}=\frac{\partial}{\partial X^{i j}} \\
D_{i}^{+}=\frac{\partial}{\partial \bar{Q}^{i}}+\frac{1}{2} Q^{k} D_{k i} \\
D_{i}^{-}=\frac{\partial}{\partial Q^{i}}-\frac{1}{2} \bar{Q}^{k} D_{k i} \\
D^{++}=Z^{i} \frac{\partial}{\partial \bar{Z}^{i}}+Q^{i} \frac{\partial}{\partial \bar{Q}^{i}} \\
D^{--}=\bar{Z}^{i} \frac{\partial}{\partial Z^{i}}+\bar{Q}^{i} \frac{\partial}{\partial Q^{i}} \\
D^{0}=\frac{\partial}{2}\left(Z^{i} \frac{\partial}{\partial Z^{i}}+Q^{i} \frac{\partial}{\partial Q^{i}}-\bar{Z}^{i} \frac{\partial}{\partial \bar{Z}^{i}}-\bar{Q}^{i} \frac{\partial}{\partial \bar{Q}^{i}}\right)
\end{gathered}
$$

The commutators that we shall use are

$$
\left[D^{++}, D_{i}^{+}\right]=0, \quad\left[D^{++}, D_{i}^{-}\right]=-D_{i}^{+}
$$




$$
\begin{gathered}
{\left[D^{--}, D_{i}^{-}\right]=0, \quad\left[D^{--}, D_{i}^{+}\right]=-D_{i}^{-}} \\
{\left[D^{++}, D^{--}\right]=2 D^{0}} \\
\left\{D_{i}^{+}, D_{j}^{+}\right\}=0, \quad\left\{D_{i}^{-}, D_{j}^{-}\right\}=0 \\
\left\{D_{i}^{+}, D_{j}^{-}\right\}=-D_{i j}
\end{gathered}
$$

We now introduce gauge fields for all the derivatives we have introduced except $D^{0}$, and set all field strengths associated with the above commutation relations to zero. By choosing the gauge $A_{i}^{+}=0$ as before, we find the analyticity condition $D_{i}^{+} A^{++}=0$, which implies that in $A^{++},\left(X^{i j}, \bar{Q}^{i}\right)$ enter only via the combination $X^{\prime i j}$ where $\zeta^{\prime}=\zeta+\frac{i}{2} \xi \cdot \bar{\xi}, \tilde{\zeta}^{\prime}=$ $\tilde{\zeta}+\frac{i}{2} \eta \cdot \bar{\eta}, x^{\prime \alpha \dot{\alpha}}=x^{\alpha \dot{\alpha}}+\frac{1}{2}\left(\xi^{\alpha} \bar{\eta}^{\dot{\alpha}}+\bar{\xi}^{\alpha} \eta^{\dot{\alpha}}\right) . A^{++}$determines $A^{--}, A_{i}^{-}, A_{i j}$ via $D^{++} A^{--}-$ $D^{--} A^{++}+\left[A^{++}, A^{--}\right]=0, A_{i}^{-}=D_{i}^{+} A^{--}$, and $A_{i j}=D_{i}^{+} D_{j}^{+} A^{--}$. The potential $A_{i j}$ has components $A_{\zeta}, A_{\tilde{\zeta}}$ which become $W$ and $\bar{W}$ once the $\zeta, \tilde{\zeta}, \omega$ dependence of all functions is restricted suitably. This can be done in a way consistent with the analyticity of $A^{++}$by imposing

$$
\begin{gathered}
\Omega(X, Q) \equiv-\frac{1}{8} \epsilon_{i j k l} X^{\prime i j} X^{\prime k l}=\zeta^{\prime} \tilde{\zeta}^{\prime}+x^{\prime \mu} x^{\prime \mu}=0 \\
Z^{i} X_{i j}^{\prime}=0
\end{gathered}
$$

where $X_{i j}^{\prime}=\frac{1}{2} \epsilon_{i j k l} X^{\prime k l}$. (Actually $(24 b)$ requires (24a) for consistency, so there is some harmless redundancy here. Equation (24b), via the Klein correspondence, can be interpreted as the realisation of the set of projective lines in $\mathbf{C} P^{3}$ as a quadric in $\mathbf{C} P^{5}[6],[7]$.) These equations are invariant under scaling of $X^{\prime i j}$ as expected for equations on $\mathbf{C} P^{5}$. One can obtain Minkowski space from the quadric (24a) by choice of an infinity twistor. If we set

$$
I=\left[\begin{array}{cc}
i \epsilon & 0 \\
0 & 0
\end{array}\right]
$$

and remove the points $I^{k l} X_{k l}^{\prime}=0$ from the quadric, then on the remainder of the quadric, by virtue of the scaling symmetry we can set $I^{k l} X_{k l}^{\prime}=2$, or $\tilde{\zeta}^{\prime}=1$. This choice breaks the symmetry between $\alpha$ and $\dot{\alpha}$, leaving only a Poincaré invariant structure, as expected for Minkowski space. Also in this case, $(24 b)$ gives the standard identification $\omega^{\alpha}=$ $x^{\prime \alpha \dot{\alpha}} \pi_{\dot{\alpha}}$. Thus on restriction to the quadric $(24), A^{++}\left(X^{\prime}, Q, Z, \bar{Z}\right)$ becomes a function of 
$x^{\prime \alpha \dot{\alpha}}, \pi^{\dot{\alpha}}, \bar{\pi}^{\dot{\alpha}}, \xi^{\alpha}, \eta^{\dot{\alpha}}$, and $D^{++}, D^{--}, D^{0}, D_{i}^{+}, D_{i}^{-}$reproduce the derivatives $(1 b)-(1 h)$. The potentials $A_{\zeta}, A_{\tilde{\zeta}}$ become $W, \bar{W}$ respectively, and so the Lagrangian for the Witten theory is $\operatorname{Tr}\left(A_{\zeta}^{2}\right)$. From the definition of $\Omega$ we see that $\frac{\partial}{\partial \zeta^{\prime}}=\tilde{\zeta}^{\prime} \frac{\partial}{\partial \Omega}$ (By this we understand that if we were to replace the variable $\zeta^{\prime}$ in our formulation by the variable $\Omega$ this is how we would rewrite the derivative $\left.\frac{\partial}{\partial \zeta^{\prime}}\right)$. Thus a general definition of the Lagrangian is $\operatorname{Tr}\left(A_{\Omega}{ }^{2}\right)$, $A_{\Omega}$ being the gauge potential in the direction $\frac{\partial}{\partial \Omega}$, i.e. the potential along the normal to the quadric $(24 a)$. For the choice $\tilde{\zeta}^{\prime}=1$ this general Lagrangian reproduces $\operatorname{Tr}\left(W^{2}\right)$; the choice $\zeta^{\prime}=1$, corresponding to an infinity twistor

$$
\tilde{I}=\left[\begin{array}{cc}
0 & 0 \\
0 & i \epsilon
\end{array}\right]
$$

leads to $\operatorname{Tr}\left(\bar{W}^{2}\right)$, which is the Lagrangian for the theory appropriate to anti-instantons with $\left(F_{\mu \nu}+\tilde{F}_{\mu \nu}\right)^{2}$ in the action (obtained from $N=2$ theory by identifying $S U(2)_{I}$ with the component $S U(2)_{L}$ of the Lorentz group). We must finally, of course, restrict to the subspace of real $x^{\mu}$ in the quadric (24).

Although we have until now emphasized the flat space formulation, the $\mathbf{C} P^{5}$ approach extends naturally to all conformally flat spacetimes. Such spaces can be obtained by different choices of the infinity twistor $I$ and removing $I \cdot X$ from the quadric (24) [6]. The complete topological invariance, under arbitrary variations of the metric, is still not manifest in this approach; nevertheless we expect it to be a fruitful perspective on the theory.

\section{References}

[1] E.Witten, Comm. Math. Phys. 117 (1988) 353.

[2] P.Braam, lectures given at the Fifth Annual University of California Summer School on Nonlinear Science, Physics and Geometry (June 1989).

[3] L.Baulieu and I.M.Singer, Topological Yang Mills Symmetry, LPTHE preprint 88/18, LPTENS preprint 88/15, talk given at the Annecy meeting on Conformal Field Theory and Related Topics (March 1988). J.M.F.Labastida and M.Pernici, Phys. Lett. B212 (1988) 56. R.Brooks, D.Montano and J.Sonnenschein, Phys. Lett. B214 (1988) 91. 
[4] A.Galperin, E.Ivanov, S.Kalitzin, V.Ogievetsky and E.Sokatchev, Class. Quantum Grav. 1 (1984) 469.

[5] J.Horne, Nucl. Phys. B318 (1989) 22.

[6] For general background on twistor theory see R.Penrose and W.Rindler, Spinors and Spacetime (Vols.1 and 2), Cambridge University Press $(1984,1986)$ and R.O.Wells, Jr., Complex Geometry in Mathematical Physics, Séminaire de Mathématiques Supérieures No.78, Les Presses de l'Université de Montréal (1982).

[7] L.P.Hughston and T.R.Hurd, Phys. Rep. 100 (1983) 273.

[8] R.Penrose, Twistor Newsletter 27 (1988) 1.

[9] R.S.Ward, Phys. Lett. 61A (1977) 81.

[10] R.Grimm, M.Sohnius and J.Wess, Nucl. Phys. B133 (1978) 275.

[11] B.M.Zupnik, Phys. Lett. B183 (1987) 175.

[12] A.Ferber, Nucl. Phys. B132 (1978) 55. 